\title{
Approximation of Solutions for Second-Order $m$-Point Nonlocal Boundary Value Problems via the Method of Generalized Quasilinearization
}

\begin{abstract}
Ahmed Alsaedi
Department of Mathematics, Faculty of Science, King Abdulaziz University, P.O. Box 80203, Jeddah 21589, Saudi Arabia

Correspondence should be addressed to Ahmed Alsaedi, aalsaedi@hotmail.com

Received 11 May 2010; Revised 29 July 2010; Accepted 2 October 2010

Academic Editor: Gennaro Infante

Copyright (C) 2011 Ahmed Alsaedi. This is an open access article distributed under the Creative Commons Attribution License, which permits unrestricted use, distribution, and reproduction in any medium, provided the original work is properly cited.

We discuss the existence and uniqueness of the solutions of a second-order $m$-point nonlocal boundary value problem by applying a generalized quasilinearization technique. A monotone sequence of solutions converging uniformly and quadratically to a unique solution of the problem is presented.
\end{abstract}

\section{Introduction}

The monotone iterative technique coupled with the method of upper and lower solutions [1-7] manifests itself as an effective and flexible mechanism that offers theoretical as well as constructive existence results in a closed set, generated by the lower and upper solutions. In general, the convergence of the sequence of approximate solutions given by the monotone iterative technique is at most linear $[8,9]$. To obtain a sequence of approximate solutions converging quadratically, we use the method of quasilinearization [10]. This method has been developed for a variety of problems [11-20]. In view of its diverse applications, this approach is quite an elegant and easier for application algorithms.

The subject of multipoint nonlocal boundary conditions, initiated by Bicadze and Samarski1 [21], has been addressed by many authors, for instance, [22-32]. The multipoint boundary conditions appear in certain problems of thermodynamics, elasticity and wave propagation, see [23] and the references therein. The multipoint boundary conditions may be understood in the sense that the controllers at the endpoints dissipate or add energy according to censors located at intermediate positions. 
In this paper, we develop the method of generalized quasilinearization to obtain a sequence of approximate solutions converging monotonically and quadratically to a unique solution of the following second-order $m$-point nonlocal boundary value problem

$$
\begin{gathered}
-x^{\prime \prime}(t)=f\left(t, x(t), x^{\prime}(t)\right), \quad t \in[0,1] \\
p x(0)-q x^{\prime}(0)=\sum_{i=1}^{m-2} \tau_{i} x\left(\eta_{i}\right), \quad p x(1)+q x^{\prime}(1)=\sum_{i=1}^{m-2} \sigma_{i} x\left(\eta_{i}\right), \quad \eta_{i} \in(0,1),
\end{gathered}
$$

where $f:[0,1] \times \mathbb{R} \times \mathbb{R} \rightarrow \mathbb{R}$ is continuous and $\tau_{i}, \sigma_{i}(i=1,2, \ldots, m-2)$ are nonnegative real constants such that $\sum_{i=1}^{m-2} \tau_{i}<1, \sum_{i=1}^{m-2} \sigma_{i}<1$, and $p, q>0$ with $p>1$.

Here we remark that [26] studies (1.1) with the boundary conditions of the form

$$
\delta x(0)-\gamma x^{\prime}(0)=0, \quad x(1)=\sum_{i=1}^{m-2} \alpha_{i} x\left(\eta_{i}\right), \quad \eta_{i} \in(0,1) .
$$

A perturbed integral equation equivalent to the problem (1.1) and (1.3) considered in [26] is

$$
x(t)=\int_{0}^{1} k(t, s) f\left(s, x(s), x^{\prime}(s)\right) d s+\left(\sum_{i=1}^{m-2} \alpha_{i} x\left(\eta_{i}\right)\right) t^{2},
$$

where

$$
k(t, s)=\frac{1}{(\delta+\gamma)} \begin{cases}(\gamma+\delta t)(1-s), & 0 \leq t \leq \mathrm{s} \\ (\delta+\gamma s)(1-t), & s \leq t \leq 1\end{cases}
$$

It can readily be verified that the solution given by (1.4) does not satisfy (1.1). On the other hand, by Green's function method, a unique solution of the problem (1.1) and (1.3) is

$$
x(t)=\int_{0}^{1} k(t, s) f\left(s, x(s), x^{\prime}(s)\right) d s+\left(\sum_{i=1}^{m-2} \alpha_{i} x\left(\eta_{i}\right)\right) \frac{\gamma+\delta t}{\delta+\gamma},
$$

where $k(t, s)$ is given by (1.5). Thus, (1.6) represents the correct form of the solution for the problem (1.1) and (1.3). 


\section{Preliminaries}

For $x \in C^{1}[0,1]$, we define $\|x\|_{1}=\|x\|+\left\|x^{\prime}\right\|$, where $\|x\|=\max \{|x(t)|: t \in[0,1]\}$. It can easily be verified that the homogeneous problem associated with (1.1)-(1.2) has only the trivial solution. Therefore, by Green's function method, the solution of (1.1)-(1.2) can be written as

$$
\begin{aligned}
x(t)= & \int_{0}^{1} G(t, s) f\left(s, x(s), x^{\prime}(s)\right) d s+\left(\sum_{i=1}^{m-2} \tau_{i} x\left(\eta_{i}\right)\right)\left(\frac{-t}{2 q+p}+\frac{q+p}{p(2 q+p)}\right) \\
& +\left(\sum_{i=1}^{m-2} \sigma_{i} x\left(\eta_{i}\right)\right)\left(\frac{t}{2 q+p}+\frac{q}{p(2 q+p)}\right),
\end{aligned}
$$

where $G(t, s)$ is the Green's function and is given by

$$
G(t, s)=\frac{1}{p(p+2 q)} \begin{cases}(q+p t)(q+p(1-s)), & 0 \leq t \leq \mathrm{s} \\ (q+p s)(q+p(1-t)), & \mathrm{s} \leq t \leq 1 .\end{cases}
$$

Note that $G(t, s)>0$ on $[0,1] \times[0,1]$.

We say that $\alpha \in C^{2}[0,1]$ is a lower solution of the boundary value problem (1.1) and (1.2) if

$$
\begin{gathered}
-\alpha^{\prime \prime}(t) \leq f\left(t, \alpha(t), \alpha^{\prime}(t)\right), \quad t \in[0,1], \\
p \alpha(0)-q \alpha^{\prime}(0) \leq \sum_{i=1}^{m-2} \tau_{i} \alpha\left(\eta_{i}\right), \quad p \alpha(1)+q \alpha^{\prime}(1) \leq \sum_{i=1}^{m-2} \sigma_{i} \alpha\left(\eta_{i}\right),
\end{gathered}
$$

and $\beta \in C^{2}[0,1]$ is an upper solution of (1.1) and (1.2) if

$$
\begin{gathered}
-\beta^{\prime \prime}(t) \geq f\left(t, \beta(t), \beta^{\prime}(t)\right), \quad t \in[0,1], \\
p \beta(0)-q \beta^{\prime}(0) \geq \sum_{i=1}^{m-2} \tau_{i} \beta\left(\eta_{i}\right), \quad p \beta(1)+q \beta^{\prime}(1) \geq \sum_{i=1}^{m-2} \sigma_{i} \beta\left(\eta_{i}\right) .
\end{gathered}
$$

Definition 2.1. A continuous function $h:[0, \infty) \rightarrow[0, \infty)$ is called a Nagumo function if

$$
\int_{\lambda}^{\infty} \frac{s d s}{h(s)}=\infty
$$

for $\lambda \geq 0$. We say that $f \in C[[0,1] \times \mathbb{R} \times \mathbb{R}]$ satisfies a Nagumo condition on $[0,1]$ relative to $\alpha, \beta$ if for every $t \in[0,1]$ and $x \in\left[\min _{t \in[0,1]} \alpha(t), \max _{t \in[0,1]} \beta(t)\right]$, there exists a Nagumo function $h$ such that $\left|f\left(t, x, x^{\prime}\right)\right| \leq h\left(\left|x^{\prime}\right|\right)$.

We need the following result [33] to establish the main result. 
Theorem 2.2. Let $f:[0,1] \times \mathbb{R}^{2} \rightarrow \mathbb{R}$ be a continuous function satisfying the Nagumo condition on $E=\left\{(t, x, y) \in[0,1] \times \mathbb{R}^{2}: \alpha \leq x \leq \beta\right\}$ where $\alpha, \beta:[0,1] \rightarrow \mathbb{R}$ are continuous functions such that $\alpha(t) \leq \beta(t)$ for all $t \in[0,1]$. Then there exists a constant $M>0$ (depending only on $\alpha, \beta$, the Nagumo function $h$ ) such that every solution $x$ of (1.1)-(1.2) with $\alpha(t) \leq x(t) \leq \beta(t), t \in[0,1]$ satisfies $\left|x^{\prime}\right| \leq M$.

If $\alpha, \beta \in C^{2}[0,1]$ are assumed to be lower and upper solutions of (1.1)-(1.2), respectively, in the statement of Theorem 2.2, then there exists a solution, $x(t)$ of (1.1) and (1.2) such that $\alpha(t) \leq x(t) \leq \beta(t), t \in[0,1]$.

Theorem 2.3. Assume that $\alpha, \beta \in C^{2}[0,1]$ are, respectively, lower and upper solutions of (1.1)-(1.2). If $f(t, x, y) \in C([0,1] \times \mathbb{R} \times \mathbb{R})$ is decreasing in $x$ for each $(t, y) \in[0,1] \times \mathbb{R}$, then $\alpha \leq \beta$ on $[0,1]$.

Proof. Let us define $u(t)=\alpha(t)-\beta(t)$ so that $u \in C^{2}([0,1])$ and satisfies the boundary conditions

$$
p u(0)-q u^{\prime}(0) \leq \sum_{i=1}^{m-2} \tau_{i} u\left(\eta_{i}\right), \quad p u(1)+q u^{\prime}(1) \leq \sum_{i=1}^{m-2} \sigma_{i} u\left(\eta_{i}\right) .
$$

For the sake of contradiction, let $u$ have a positive maximum at some $t_{0} \in[0,1]$. If $t_{0} \in(0,1)$, then $u^{\prime}\left(t_{0}\right)=0$ and $u^{\prime \prime}\left(t_{0}\right) \leq 0$. On the other hand, in view of the decreasing property of $f(t, x, y)$ in $x$, we have

$$
u^{\prime \prime}\left(t_{0}\right)=\alpha^{\prime \prime}\left(t_{0}\right)-\beta^{\prime \prime}\left(t_{0}\right) \geq-f\left(t_{0}, \alpha\left(t_{0}\right), \alpha^{\prime}\left(t_{0}\right)\right)+f\left(t_{0}, \beta\left(t_{0}\right), \beta^{\prime}\left(t_{0}\right)\right)>0,
$$

which is a contradiction. If we suppose that $u$ has a positive maximum at $t_{0}=0$, then it follows from the first of boundary conditions (2.6) that

$$
p u(0)-q u^{\prime}(0) \leq \sum_{i=1}^{m-2} \tau_{i} u\left(\eta_{i}\right) \leq u(0),
$$

which implies that $(p-1) u(0) \leq q u^{\prime}(0)$. Now as $p>1, q>0, u(0)>0, u^{\prime}(0) \leq 0$, therefore we obtain a contradiction. We have a similar contradiction at $t_{0}=1$. Thus, we conclude that $\alpha(t) \leq \beta(t), t \in[0,1]$.

\section{Main Results}

Theorem 3.1. Assume that

$\left(\mathrm{A}_{1}\right)$ the functions $\alpha, \beta \in C^{2}[0,1]$ are, respectively, lower and upper solutions of (1.1)-(1.2) such that $\alpha \leq \beta$ on $[0,1]$;

$\left(\mathrm{A}_{2}\right)$ the function $f \in C^{2}([0,1] \times \mathbb{R} \times \mathbb{R})$ satisfies a Nagumo condition relative to $\alpha, \beta$ and $f_{x} \leq 0$ on $[0,1] \times\left[\min _{t \in[0,1]} \alpha(t), \max _{t \in[0,1]} \beta(t)\right] \times[-M, M]$, where $M$ is a positive constant depending on $\alpha, \beta$, and the Nagumo function $h$. Further, there exists a function $\phi \in C^{2}\left([0,1] \times \mathbb{R}^{2}\right)$ such that $\Psi(f+\phi) \geq 0$ with $\Psi(\phi) \geq 0$ on $[0,1] \times$ $\left[\min _{t \in[0,1]} \alpha(t), \max _{t \in[0,1]} \beta(t)\right] \times[-M, M]$, where

$$
\Psi=(x-y)^{2} \frac{\partial^{2}}{\partial x^{2}}+2(x-y)\left(x^{\prime}-y^{\prime}\right) \frac{\partial^{2}}{\partial x \partial x^{\prime}}+\left(x^{\prime}-y^{\prime}\right)^{2} \frac{\partial^{2}}{\partial x^{\prime 2}} .
$$


Then, there exists a monotone sequence $\left\{\alpha_{n}\right\}$ of approximate solutions converging uniformly to a unique solution of the problems (1.1)-(1.2).

Proof. For $y \in \mathbb{R}$, we define $\omega(y)=\max \{-M, \min \{y, M\}\}$ and consider the following modified $m$-point BVP

$$
\begin{gathered}
-x^{\prime \prime}(t)=f\left(t, x(t), \omega\left(x^{\prime}(t)\right)\right), \quad t \in[0,1] \\
p x(0)-q x^{\prime}(0)=\sum_{i=1}^{m-2} \tau_{i} x\left(\eta_{i}\right), \quad p x(1)+q x^{\prime}(1)=\sum_{i=1}^{m-2} \sigma_{i} x\left(\eta_{i}\right) .
\end{gathered}
$$

We note that $\alpha, \beta$ are, respectively, lower and upper solutions of (3.2) and for every $(t, x) \in$ $[0,1] \times\left[\min _{t \in[0,1]} \alpha(t), \max _{t \in[0,1]} \beta(t)\right]$, we have

$$
|f| \leq h\left(\left|\omega\left(x^{\prime}\right)\right|\right)=\tilde{h}\left(\left|x^{\prime}\right|\right)
$$

where $\tilde{h}(\cdot)=h(\omega(\cdot))$. As

$$
\int_{0}^{\infty} \frac{s d s}{\widetilde{h}(s)}=\int_{0}^{M} \frac{s d s}{h(s)}+\int_{M}^{\infty} \frac{s d s}{h(M)}=\infty
$$

so $\tilde{h}$ is a Nagumo function. Furthermore, there exists a constant $N$ depending on $\alpha, \beta$, and Nagumo function $h$ such that

$$
\int_{0}^{M} \frac{s d s}{\widetilde{h}(s)} \geq \int_{0}^{N} \frac{s d s}{h(s)}>(\max \{\beta(t): t \in[0,1]\}-\min \{\alpha(t): t \in[0,1]\})
$$

where $M>\max \left\{N,\left\|\alpha^{\prime}\right\|,\left\|\beta^{\prime}\right\|\right\}$. Thus, any solution $x$ of (3.2) with $\alpha(t) \leq x(t) \leq \beta(t), t \in[0,1]$ satisfies $\left|x^{\prime}\right| \leq M$ on $[0,1]$ and hence it is a solution of (1.1)-(1.2).

Let us define a function $F:[0,1] \times \mathbb{R}^{2} \rightarrow \mathbb{R}$ by

$$
F\left(t, x, x^{\prime}\right)=f\left(t, x, x^{\prime}\right)+\phi\left(t, x, x^{\prime}-\omega\left(x^{\prime}\right)\right)
$$

In view of the assumption $\left(\mathrm{A}_{2}\right)$, it follows that $F \in C^{2}\left([0,1] \times \mathbb{R}^{2}\right)$ and satisfies $\Psi(F) \geq 0$ on $[0,1] \times\left[\min _{t \in[0,1]} \alpha(t), \max _{t \in[0,1]} \beta(t)\right] \times[-M, M]$. Therefore, by Taylor's theorem, we obtain

$$
\begin{aligned}
f\left(t, x, \omega\left(x^{\prime}\right)\right) \geq & f\left(t, y, \omega\left(y^{\prime}\right)\right)+F_{x}\left(t, y, \omega\left(y^{\prime}\right)\right)(x-y) \\
& +F_{x^{\prime}}\left(t, y, \omega\left(y^{\prime}\right)\right)\left(\omega\left(x^{\prime}\right)-\omega\left(y^{\prime}\right)\right)-[\phi(t, x, 0)-\phi(t, y, 0)] \\
\geq & f\left(t, y, \omega\left(y^{\prime}\right)\right)+\left[F_{x}\left(t, y, \omega\left(y^{\prime}\right)\right)-\phi_{x}(t, \beta, 0)\right](x-y) \\
& +F_{x^{\prime}}\left(t, y, \omega\left(y^{\prime}\right)\right)\left(\omega\left(x^{\prime}\right)-\omega\left(y^{\prime}\right)\right) .
\end{aligned}
$$


We set

$$
\begin{aligned}
H\left(t, x, x^{\prime} ; y, y^{\prime}\right)= & f\left(t, y, \omega\left(y^{\prime}\right)\right)+\left[F_{x}\left(t, y, \omega\left(y^{\prime}\right)\right)-\phi_{x}(t, \beta, 0)\right](x-y) \\
& +F_{x^{\prime}}\left(t, y, \omega\left(y^{\prime}\right)\right)\left(\omega\left(x^{\prime}\right)-\omega\left(y^{\prime}\right)\right)
\end{aligned}
$$

and observe that

$$
\begin{aligned}
& f\left(t, x, \omega\left(x^{\prime}\right)\right) \geq H\left(t, x, x^{\prime} ; y, y^{\prime}\right), \\
& f\left(t, x, \omega\left(x^{\prime}\right)\right)=H\left(t, x, x^{\prime} ; x, x^{\prime}\right) .
\end{aligned}
$$

By the mean value theorem, we can find $\alpha \leq c_{1} \leq y$ and $\alpha^{\prime} \leq c_{2} \leq y^{\prime}\left(c_{1}, c_{2}\right.$ depend on $y, y^{\prime}$, resp.), such that

$$
f\left(t, y, \omega\left(y^{\prime}\right)\right)-f\left(t, \alpha(t), \alpha^{\prime}(t)\right)=f_{x}\left(t, c_{1}, c_{2}\right)(y-\alpha(t))+f_{x^{\prime}}\left(t, c_{1}, c_{2}\right)\left(\omega\left(y^{\prime}\right)-\alpha^{\prime}(t)\right) .
$$

\section{Letting}

$$
H_{1}\left(t, x, x^{\prime} ; y, y^{\prime}\right)=f\left(t, \alpha(t), \alpha^{\prime}(t)\right)+f_{x}\left(t, c_{1}, c_{2}\right)(x-\alpha(t))+f_{x^{\prime}}\left(t, c_{1}, c_{2}\right)\left(\omega\left(x^{\prime}\right)-\alpha^{\prime}(t)\right)
$$

we note that

$$
\begin{gathered}
f\left(t, y, \omega\left(y^{\prime}\right)\right)=H_{1}\left(t, y, y^{\prime} ; y, y^{\prime}\right) \\
f\left(t, \alpha(t), \alpha^{\prime}(t)\right)=H_{1}\left(t, \alpha(t), \alpha^{\prime}(t) ; y, y^{\prime}\right) .
\end{gathered}
$$

Let us define $\widetilde{H}$ as

$$
\widetilde{H}= \begin{cases}H\left(t, x, x^{\prime} ; y, y^{\prime}\right), & \text { for } x \geq y \\ H_{1}\left(t, x, x^{\prime} ; y, y^{\prime}\right), & \text { for } x \leq y\end{cases}
$$

Clearly $\widetilde{H}$ is continuous and bounded on $[0,1] \times\left[\min _{t \in[0,1]} \alpha(t), \max _{t \in[0,1]} \beta(t)\right] \times \mathbb{R}$ and satisfies a Nagumo condition relative to $\alpha, \beta$. For every $\alpha(t) \leq y \leq \beta(t)$ and $y^{\prime} \in \mathbb{R}$, we consider the m-point BVP

$$
\begin{gathered}
-x^{\prime \prime}=\widetilde{H}\left(t, x, x^{\prime} ; y, y^{\prime}\right), \quad t \in[0,1] \\
p x(0)-q x^{\prime}(0)=\sum_{i=1}^{m-2} \tau_{i} x\left(\eta_{i}\right), \quad p x(1)+q x^{\prime}(1)=\sum_{i=1}^{m-2} \sigma_{i} x\left(\eta_{i}\right) .
\end{gathered}
$$


Using (3.9), (3.12) and (3.13), we have

$$
\begin{gathered}
\widetilde{H}\left(t, \alpha(t), \alpha^{\prime}(t) ; y, y^{\prime}\right)=H_{1}\left(t, \alpha(t), \alpha^{\prime}(t) ; y, y^{\prime}\right)=f\left(t, \alpha(t), \alpha^{\prime}(t)\right) \geq-\alpha^{\prime \prime}(t), \\
p \alpha(0)-q \alpha^{\prime}(0) \leq \sum_{i=1}^{m-2} \tau_{i} \alpha\left(\eta_{i}\right), \quad p \alpha(1)+q \alpha^{\prime}(1) \leq \sum_{i=1}^{m-2} \sigma_{i} \alpha\left(\eta_{i}\right), \\
\widetilde{H}\left(t, \beta(t), \beta^{\prime}(t) ; y, y^{\prime}\right)=H\left(t, \beta(t), \beta^{\prime}(t) ; y, y^{\prime}\right) \leq f\left(t, \beta(t), \beta^{\prime}(t)\right) \leq-\beta^{\prime \prime}(t), \\
p \beta(0)-q \beta^{\prime}(0) \geq \sum_{i=1}^{m-2} \tau_{i} \beta\left(\eta_{i}\right), \quad p \beta(1)+q \beta^{\prime}(1) \geq \sum_{i=1}^{m-2} \sigma_{i} \beta\left(\eta_{i}\right) .
\end{gathered}
$$

Thus, $\alpha, \beta$ are lower and upper solutions of (3.14), respectively. Since $\widetilde{H}$ satisfies a Nagumo condition, there exists a constant $M_{1}>\max \left\{\left\|\alpha^{\prime}\right\|,\left\|\beta^{\prime}\right\|\right\}$ (depending on $\alpha, \beta$ and a Nagumo function) such that any solution $x$ of (3.14) with $\alpha(t) \leq x(t) \leq \beta(t)$ satisfies $\left|x^{\prime}\right|<M_{1}$ on $[0,1]$.

Now, we choose $\alpha_{0}=\alpha$ and consider the problem

$$
\begin{gathered}
-x^{\prime \prime}=\widetilde{H}\left(t, x, x^{\prime} ; \alpha_{0}, \alpha_{0}^{\prime}\right), \quad t \in[0,1] \\
p x(0)-q x^{\prime}(0)=\sum_{i=1}^{m-2} \tau_{i} x\left(\eta_{i}\right), \quad p x(1)+q x^{\prime}(1)=\sum_{i=1}^{m-2} \sigma_{i} x\left(\eta_{i}\right) .
\end{gathered}
$$

Using $\left(A_{1}\right),(3.9),(3.12)$ and (3.13), we obtain

$$
\begin{gathered}
\widetilde{H}\left(t, \alpha_{0}, \alpha_{0}^{\prime} ; \alpha_{0}, \alpha_{0}\right)=f\left(t, \alpha_{0}, \alpha_{0}^{\prime}\right) \geq-\alpha_{0}^{\prime \prime}(t), \\
p \alpha_{0}(0)-q \alpha_{0}^{\prime}(0) \leq \sum_{i=1}^{m-2} \tau_{i} \alpha_{0}\left(\eta_{i}\right), \quad p \alpha_{0}(1)+q \alpha_{0}^{\prime}(1) \leq \sum_{i=1}^{m-2} \sigma_{i} \alpha_{0}\left(\eta_{i}\right), \\
\widetilde{H}\left(t, \beta(t), \beta^{\prime}(t) ; \alpha_{0}, \alpha_{0}^{\prime}\right)=H\left(t, \beta(t), \beta^{\prime}(t) ; \alpha_{0}, \alpha_{0}^{\prime}\right) \leq f\left(t, \beta(t), \beta^{\prime}(t)\right) \leq-\beta^{\prime \prime}(t), \\
p \beta(0)-q \beta^{\prime}(0) \geq \sum_{i=1}^{m-2} \tau_{i} \beta\left(\eta_{i}\right), \quad p \beta(1)+q \beta^{\prime}(1) \geq \sum_{i=1}^{m-2} \sigma_{i} \beta\left(\eta_{i}\right),
\end{gathered}
$$

which imply that $\alpha_{0}$ and $\beta$ are lower and upper solutions of (3.16). Hence by Theorems 2.2 and 2.3, there exists a unique solution $\alpha_{1}$ of (3.16) such that

$$
\alpha_{0} \leq \alpha_{1} \leq \beta(t), \quad\left|\alpha_{1}^{\prime}\right| \leq M_{1}, \quad t \in[0,1]
$$


Note that the uniqueness of the solution follows by Theorem 2.3. Using (3.9) and (3.13) together with the fact that $\alpha_{1}$ is solution of (3.16), we find that $\alpha_{1}$ is a lower solution of (3.2), that is,

$$
\begin{gathered}
-\alpha_{1}^{\prime \prime}=\widetilde{H}\left(t, \alpha_{1}, \alpha_{1}^{\prime} ; \alpha_{0}, \alpha_{0}^{\prime}\right) \leq f\left(t, \alpha_{1}, \omega\left(\alpha_{1}^{\prime}\right)\right), \quad t \in[0,1] \\
p \alpha_{1}(0)-q \alpha_{1}^{\prime}(0)=\sum_{i=1}^{m-2} \tau_{i} \alpha_{1}\left(\eta_{i}\right), \quad p \alpha_{1}(1)+q \alpha_{1}^{\prime}(1)=\sum_{i=1}^{m-2} \sigma_{i} \alpha_{1}\left(\eta_{i}\right) .
\end{gathered}
$$

In a similar manner, it can be shown by using $\left(\mathrm{A}_{1}\right),(3.12),(3.13)$, and (3.19) that $\alpha_{1}$ and $\beta$ are lower and upper solutions of the following $m$-point BVP

$$
\begin{gathered}
-x^{\prime \prime}=\widetilde{H}\left(t, x, x^{\prime} ; \alpha_{1}, \alpha_{1}^{\prime}\right), \quad t \in[0,1], \\
p x(0)-q x^{\prime}(0)=\sum_{i=1}^{m-2} \tau_{i} x\left(\eta_{i}\right), \quad p x(1)+q x^{\prime}(1)=\sum_{i=1}^{m-2} \sigma_{i} x\left(\eta_{i}\right) .
\end{gathered}
$$

Again, by Theorems 2.2 and 2.3, there exists a unique solution $\alpha_{2}$ of (3.20) such that

$$
\alpha_{1}(t) \leq \alpha_{2}(t) \leq \beta(t), \quad\left|\alpha_{2}^{\prime}(t)\right| \leq M_{1}, \quad t \in[0,1] .
$$

Continuing this process successively, we obtain a bounded monotone sequence $\left\{\alpha_{n}\right\}$ of solutions satisfying

$$
\alpha_{1}(t) \leq \alpha_{2}(t) \leq \alpha_{3}(t) \leq \cdots \leq \alpha_{n}(t) \leq \beta(t), \quad t \in[0,1],
$$

where $\alpha_{n}$ is a solution of the problem

$$
\begin{aligned}
-x^{\prime \prime} & =\widetilde{H}\left(t, x, x^{\prime} ; \alpha_{n-1}, \alpha_{n-1}^{\prime}\right), \quad t \in[0,1], \\
p x(0)-q x^{\prime}(0) & =\sum_{i=1}^{m-2} \tau_{i} x\left(\eta_{i}\right), \quad p x(1)+q x^{\prime}(1)=\sum_{i=1}^{m-2} \sigma_{i} x\left(\eta_{i}\right),
\end{aligned}
$$

and is given by

$$
\begin{aligned}
x(t)= & \int_{0}^{1} G(t, s) \widetilde{H}\left(s, \alpha_{n}, \alpha_{n}^{\prime} ; \alpha_{n-1}, \alpha_{n-1}^{\prime}\right) d s+\left(\sum_{i=1}^{m-2} \tau_{i} x\left(\eta_{i}\right)\right)\left(\frac{-t}{2 q+p}+\frac{q+p}{p(2 q+p)}\right) \\
& +\left(\sum_{i=1}^{m-2} \sigma_{i} x\left(\eta_{i}\right)\right)\left(\frac{t}{2 q+p}+\frac{q}{p(2 q+p)}\right) .
\end{aligned}
$$

Since $\widetilde{H}$ is bounded on $[0,1] \times\left[\min _{t \in[0,1]} \alpha(t), \max _{t \in[0,1]} \beta(t)\right] \times \mathbb{R} \times\left[\min _{t \in[0,1]} \alpha(t)\right.$, $\left.\max _{t \in[0,1]} \beta(t)\right] \times \mathbb{R}$, therefore it follows that the sequences $\left\{\alpha_{n}^{(j)}\right\}(j=0,1)$ are uniformly bounded and equicontinuous on $[0,1]$. Hence, by Ascoli-Arzela theorem, there exist the subsequences and a function $x \in C^{1}([0,1])$ such that $\alpha_{n}^{(j)} \rightarrow x^{(j)}$ uniformly on $[0,1]$ as 
$n \rightarrow \infty$. Taking the limit $n \rightarrow \infty$, we find that $\widetilde{H}\left(t, \alpha_{n}, \alpha_{n}^{\prime} ; \alpha_{n-1}, \alpha_{n-1}^{\prime}\right) \rightarrow f\left(t, x, \omega\left(x^{\prime}\right)\right)$ which consequently yields

$$
\begin{aligned}
x(t)= & \int_{0}^{1} G(t, s) f\left(s, x(s), \omega\left(x^{\prime}(s)\right)\right) d s+\left(\sum_{i=1}^{m-2} \tau_{i} x\left(\eta_{i}\right)\right)\left(\frac{-t}{2 q+p}+\frac{q+p}{p(2 q+p)}\right) \\
& +\left(\sum_{i=1}^{m-2} \sigma_{i} x\left(\eta_{i}\right)\right)\left(\frac{t}{2 q+p}+\frac{q}{p(2 q+p)}\right) .
\end{aligned}
$$

This proves that $x$ is a solution of (3.2).

Theorem 3.2. Assume that $\left(A_{1}\right)$ and $\left(A_{2}\right)$ hold. Further, one assumes that

$\left(\mathrm{A}_{3}\right)$ the function $F \in C^{2}([0,1] \times \mathbb{R} \times \mathbb{R})$ satisfies $y\left(\partial / \partial x^{\prime}\right)\left[F(t, x, y)+m y^{2}\right] \leq 0$ for $|y| \geq$ $M$, where $m=\max \left\{\left|F_{x^{\prime} x^{\prime}}(t, x, y)\right|:(t, x, y) \in[0,1] \times\left[\min _{t \in[0,1]} \alpha(t), \max _{t \in[0,1]} \beta(t)\right] \times\right.$ $[-M, M]\}$, and $F=f+\phi$.

Then, the convergence of the sequence $\left\{\alpha_{n}\right\}$ of approximate solutions (obtained in Theorem 3.1 ) is quadratic.

Proof. Let us set $e_{n+1}(t)=x(t)-\alpha_{n+1}(t) \geq 0$ so that $e_{n+1}$ satisfies the boundary conditions

$$
p e_{n+1}(0)-q e_{n+1}^{\prime}(0)=\sum_{i=1}^{m-2} \tau_{i} e_{n+1}\left(\eta_{i}\right), \quad p e_{n+1}(1)+q e_{n+1}^{\prime}(1)=\sum_{i=1}^{m-2} \sigma_{i} e_{n+1}\left(\eta_{i}\right)
$$

In view of the assumption $\left(\mathrm{A}_{3}\right)$, for every $(t, x) \in[0,1] \times\left[\min _{t \in[0,1]} \alpha(t), \max _{t \in[0,1]} \beta(t)\right]$, it follows that

$$
F_{x^{\prime}}(t, x, M)+2 m M \leq 0, \quad F_{x^{\prime}}(t, x,-M)-2 m M \geq 0 .
$$

Now, by Taylor's theorem, we have

$$
\begin{aligned}
-e_{n+1}^{\prime \prime}(t)= & {\left[F\left(t, x, x^{\prime}\right)-\phi(t, x, 0)\right] } \\
- & {\left[f\left(t, \alpha_{n}, \omega\left(\alpha_{n}^{\prime}\right)\right)+F_{x}\left(t, \alpha, \omega\left(\alpha_{n}^{\prime}\right)\right)\left(\alpha_{n+1}-\alpha_{n}\right)\right.} \\
& \left.\quad-\phi_{x}(t, \beta, 0)\left(\alpha_{n+1}-\alpha_{n}\right)+F_{x^{\prime}}\left(t, \alpha_{n}, \omega\left(\alpha_{n}^{\prime}\right)\right)\left(\omega\left(\alpha_{n+1}^{\prime}\right)-\omega\left(\alpha_{n}^{\prime}\right)\right)\right] \\
= & F_{x}\left(t, \alpha_{n}, \omega\left(\alpha_{n}^{\prime}\right)\right)\left(x-\alpha_{n+1}\right)+F_{x^{\prime}}\left(t, \alpha_{n}, \omega\left(\alpha_{n}^{\prime}\right)\right)\left(x^{\prime}-\omega\left(\alpha_{n+1}^{\prime}\right)\right) \\
+ & \frac{1}{2}\left[\left(x-\alpha_{n}\right)^{2} F_{x x}\left(t, z_{1}, z_{2}\right)+2\left(x-\alpha_{n}\right)\left(x^{\prime}-\omega\left(\alpha_{n}^{\prime}\right)\right) F_{x x^{\prime}}\left(t, z_{1}, z_{2}\right)\right. \\
& \left.\quad+\left(x^{\prime}-\omega\left(\alpha_{n}^{\prime}\right)\right)^{2} F_{x^{\prime} x^{\prime}}\left(t, z_{1}, z_{2}\right)\right] \\
& -\left[\phi(t, x, 0)-\phi\left(t, \alpha_{n}, 0\right)-\phi_{x}(t, \beta, 0)\left(\alpha_{n+1}-\alpha_{n}\right)\right] \\
\leq & F_{x^{\prime}}\left(t, \alpha_{n}, \omega\left(\alpha_{n}^{\prime}\right)\right)\left(x^{\prime}-\omega\left(\alpha_{n+1}^{\prime}\right)\right)+\left(\frac{M_{2}}{2}\right)\left(\left|x-\alpha_{n}\right|+\left|x^{\prime}-\omega\left(\alpha_{n}^{\prime}\right)\right|\right)^{2}+\rho_{1}\left(x-\alpha_{n}\right)^{2},
\end{aligned}
$$


where $\alpha_{n} \leq z_{1} \leq x, \omega\left(\alpha_{n}^{\prime}\right) \leq z_{2} \leq x^{\prime}, \alpha_{n} \leq \xi \leq \beta, M_{2}=\max \left\{\left|F_{x x}\right|,\left|F_{x x^{\prime}}\right|,\left|F_{x^{\prime} x^{\prime}}\right|\right\}$ on $[0,1] \times\left[\min _{t \in[0,1]} \alpha(t), \max _{t \in[0,1]} \beta(t)\right] \times[-M, M]$ and $\rho_{1}=\rho \max \left\{\phi_{x x}(t, x, 0):(t, x, 0) \in\right.$ $\left.[0,1] \times\left[\min _{t \in[0,1]} \alpha(t), \max _{t \in[0,1]} \beta(t)\right]\right\}$ with $\rho>1$ satisfying $\beta-\alpha_{n} \leq \rho\left(x-\alpha_{n}\right)$ on $[0,1]$. Also, in view of (3.13), we have

$$
\begin{aligned}
-e_{n+1}^{\prime \prime}(t) & =f\left(t, x, x^{\prime}\right)-\widetilde{H}\left(t, \alpha_{n+1}, \alpha_{n+1}^{\prime} ; \alpha_{n}, \alpha_{n}^{\prime}\right) \\
& \geq f\left(t, x, x^{\prime}\right)-f\left(t, \alpha_{n+1}, \omega\left(\alpha_{n+1}^{\prime}\right)\right) \\
& =f_{x}\left(t, c_{3}, c_{4}\right) e_{n+1}+f_{x^{\prime}}\left(t, c_{3}, c_{4}\right)\left(x^{\prime}-\omega\left(\alpha_{n+1}^{\prime}\right)\right) \\
& \geq-\gamma e_{n+1}+f_{x^{\prime}}\left(t, c_{3}, c_{4}\right)\left(x^{\prime}-\omega\left(\alpha_{n+1}^{\prime}\right)\right),
\end{aligned}
$$

where $\alpha_{n+1} \leq c_{3} \leq x, \omega\left(\alpha_{n+1}^{\prime}\right) \leq c_{4} \leq x^{\prime}$ and $\gamma=\max \left\{\left|f_{x}(t, x, y)\right|:(t, x, y) \in[0,1] \times\right.$ $\left.\left[\min _{t \in[0,1]} \alpha(t), \max _{t \in[0,1]} \beta(t)\right] \times[-M, M]\right\}$.

Now we show that $\omega\left(\alpha_{n+1}^{\prime}(t)\right)=\alpha_{n+1}^{\prime}(t)$. By the mean value theorem, for every $y_{1} \in$ $[-M, M]$ and $\omega\left(\alpha_{n+1}^{\prime}(t)\right) \leq c_{5} \leq y_{1}$, we obtain

$$
F_{x^{\prime}}\left(t, \alpha_{n}(t), y_{1}\right)=F_{x^{\prime}}\left(t, \alpha_{n}(t), \omega\left(\alpha_{n+1}^{\prime}(t)\right)\right)+F_{x^{\prime} x^{\prime}}\left(t, \alpha_{n}(t), c_{5}\right)\left(y_{1}-\omega\left(\alpha_{n+1}^{\prime}(t)\right)\right) .
$$

Let $\alpha_{n+1}^{\prime}>M$ for some $t \in[0,1]$. Then $\omega\left(\alpha_{n+1}^{\prime}(t)\right)=M$ and (3.30) becomes

$$
\begin{aligned}
F_{x^{\prime}}\left(t, \alpha_{n}(t), y_{1}\right) & =F_{x^{\prime}}\left(t, \alpha_{n}(t), M\right)+F_{x^{\prime} x^{\prime}}\left(t, \alpha_{n}(t), c_{5}\right)\left(y_{1}-M\right) \\
& \leq F_{x^{\prime}}\left(t, \alpha_{n}(t), M\right)-m\left(y_{1}-M\right) .
\end{aligned}
$$

In particular, taking $y_{1}=-M$ and using (3.27), we have

$$
F_{x^{\prime}}\left(t, \alpha_{n}(t),-M\right) \leq F_{x^{\prime}}\left(t, \alpha_{n}(t), M\right)+2 m M \leq 0,
$$

which contradicts that $F_{x^{\prime}}\left(t, \alpha_{n}(t),-M\right) \geq 2 m M>0$. Similarly, letting $\alpha_{n+1}^{\prime}<-M$ for some $t \in[0,1]$, we get a contradiction. Thus, it follows that $\left|\alpha_{n+1}^{\prime}(t)\right| \leq M$ for every $t \in[0,1]$, which implies that $\omega\left(\alpha_{n+1}^{\prime}(t)\right)=\alpha_{n+1}^{\prime}(t)$ and consequently, (3.28) and (3.29) take the form

$$
-e_{n+1}^{\prime \prime}(t) \leq F_{x^{\prime}}\left(t, \alpha_{n}, \omega\left(\alpha_{n}^{\prime}(t)\right)\right) e_{n+1}^{\prime}(t)+M_{3}\left\|e_{n}\right\|_{1}^{2}
$$

where $M_{3}=\rho_{1}+\left(M_{2} / 2\right)$ and

$$
-e_{n+1}^{\prime \prime}(t) \geq-\gamma e_{n+1}(t)+f_{x^{\prime}}\left(t, c_{3}, c_{4}\right) e_{n+1}^{\prime}(t) .
$$

Now, by a comparison principle, we can obtain $e_{n+1}(t) \leq r(t)$ on $[0,1]$, where $r(t)$ is a solution of the problem

$$
\begin{gathered}
-r^{\prime \prime}(t)=F_{x^{\prime}}\left(t, \alpha_{n}, \omega\left(\alpha_{n}^{\prime}(t)\right)\right) r^{\prime}(t)+M_{3}\left\|e_{n}\right\|_{1}^{2} \\
p r(0)-q r^{\prime}(0)=\sum_{i=1}^{m-2} \tau_{i} e_{n+1}\left(\eta_{i}\right), \quad p r(1)+q r^{\prime}(1)=\sum_{i=1}^{m-2} \sigma_{i} e_{n+1}\left(\eta_{i}\right) .
\end{gathered}
$$


Since $F_{x^{\prime}}$ is continuous and bounded on $[0,1] \times\left[\min _{t \in[0,1]} \alpha(t), \max _{t \in[0,1]} \beta(t)\right] \times \mathbb{R}$, there exist $\zeta_{2}, \zeta_{1}>0$ (independent of $n$ ) such that $-\zeta_{1} \leq F_{x^{\prime}} \leq \zeta_{2}$ on $[0,1] \times\left[\min _{t \in[0,1]} \alpha(t), \max _{t \in[0,1]} \beta(t)\right] \times$ $[-M, M]$. Since $\zeta_{2}-F_{x^{\prime}}\left(t, \alpha_{n}, \omega\left(\alpha_{n}^{\prime}\right)\right) \geq 0$ on $[0,1]$, so we can rewrite (3.35) as

$$
\begin{gathered}
r^{\prime \prime}(t)+\zeta_{2} r^{\prime}(t)=\left(\zeta_{2}-F_{x^{\prime}}\left(t, \alpha_{n}, \omega\left(\alpha_{n}^{\prime}\right)\right)\right) r^{\prime}(t)-M_{3}\left\|e_{n}\right\|_{1}^{2} \\
p r(0)-q r^{\prime}(0)=\sum_{i=1}^{m-2} \tau_{i} e_{n+1}\left(\eta_{i}\right), \quad p r(1)+q r^{\prime}(1)=\sum_{i=1}^{m-2} \sigma_{i} e_{n+1}\left(\eta_{i}\right),
\end{gathered}
$$

whose solution is given by

$$
\begin{aligned}
r(t)= & \int_{0}^{1} G_{\zeta_{2}}(t, s)\left(\left(\zeta_{2}-F_{x^{\prime}}\left(t, \alpha_{n}, \omega\left(\alpha_{n}^{\prime}\right)\right)\right) r^{\prime}(s)-M_{3}\left\|e_{n}\right\|_{1}^{2}\right) d s \\
& +\left(\sum_{i=1}^{m-2} \tau_{i} e_{n+1}\left(\eta_{i}\right)\right)\left(\frac{-t}{2 q+p}+\frac{q+p}{p(2 q+p)}\right)+\left(\sum_{i=1}^{m-2} \sigma_{i} e_{n+1}\left(\eta_{i}\right)\right)\left(\frac{t}{2 q+p}+\frac{q}{p(2 q+p)}\right)
\end{aligned}
$$

where

$$
G_{\zeta_{2}}(t, s)=\frac{-1}{\zeta_{2}\left(\left(p+q \zeta_{2}\right) / p-e^{-\zeta_{2}}\right)} \begin{cases}\left(1-\frac{p+\zeta_{2} q}{p} e^{-\zeta_{2}(1-s)}\right)\left(\frac{p+\zeta_{2} q}{p}-e^{-\zeta_{2} t}\right), & 0 \leq t \leq \mathrm{s}, \\ \left(e^{-\zeta_{2}(t-s)}-\frac{p+\zeta_{2} q}{p} e^{-\zeta_{2}(1-s)}\right)\left(\frac{p+\zeta_{2} q}{p}-e^{-\zeta_{2} s}\right), & \mathrm{s} \leq t \leq 1,\end{cases}
$$

Introducing the integrating factor $\mu(t)=e^{\int_{0}^{t} F_{x^{\prime}}\left(s, \alpha_{n}(s), \omega\left(\alpha_{n}^{\prime}(s)\right)\right) d s}$ such that $e^{-\zeta_{1} t}<\mu \leq e^{\zeta_{2} t}$, (3.34) takes the form

$$
\left(r^{\prime}(t) \mu(t)\right)^{\prime}=-M_{3}\left\|e_{n}\right\|_{1}^{2} \mu(t)
$$

Integrating (3.39) from 0 to $t$ and using $r^{\prime}(0) \geq(-1 / q) \sum_{i=1}^{m-2} \tau_{i} e_{n+1}\left(\eta_{i}\right)$, we obtain

$$
r^{\prime}(t) \mu(t) \geq \frac{-1}{q} \sum_{i=1}^{m-2} \tau_{i} e_{n+1}\left(\eta_{i}\right)-M_{3}\left\|e_{n}\right\|_{1}^{2} \int_{0}^{t} \mu(s) d s,
$$

which can alternatively be written as

$$
\begin{aligned}
r^{\prime}(t) & \geq \frac{-1}{q e^{\zeta_{1} 1}} \sum_{i=1}^{m-2} \tau_{i} e_{n+1}\left(\eta_{i}\right)-\frac{M_{3}}{\zeta_{2} e^{\zeta_{1} t}}\left\|e_{n}\right\|_{1}^{2}\left(e^{\zeta_{2}}-1\right) \\
& \geq \frac{-1}{q} \sum_{i=1}^{m-2} \tau_{i}\left\|e_{n+1}\right\|-\frac{M_{3}}{\zeta_{2}}\left\|e_{n}\right\|_{1}^{2}\left(e^{\zeta_{2}}-1\right)=-\rho_{1}\left\|e_{n+1}\right\|-\rho_{2}\left\|e_{n}\right\|_{1}^{2}
\end{aligned}
$$


where $\rho_{1}=(1 / q) \sum_{i=1}^{m-2} \tau_{i}, \rho_{2}=\left(M_{3} / \zeta_{2}\right)\left(e^{\zeta_{2}}-1\right)$. Using the fact that $G_{\zeta_{2}}(t, s) \leq 0$ together with (3.41) yields

$$
\begin{aligned}
G_{\zeta_{2}}(t, s)\left(\zeta_{2}-F_{x^{\prime}}\right) r^{\prime}(t) & \leq\left|G_{\zeta_{2}}(t, s)\right|\left(\zeta_{2}-F_{x^{\prime}}\right)\left(\rho_{1}\left\|e_{n+1}\right\|+\rho_{2}\left\|e_{n}\right\|_{1}^{2}\right) \\
& \leq\left|G_{\zeta_{2}}(t, s)\right|\left(\zeta_{2}+\zeta_{1}\right)\left(\rho_{1}\left\|e_{n+1}\right\|+\rho_{2}\left\|e_{n}\right\|_{1}^{2}\right)
\end{aligned}
$$

which, on substituting in (3.37), yields

$$
\begin{aligned}
e_{n+1} \leq & r(t) \leq \int_{0}^{1}\left|G_{\zeta_{2}}(t, s)\right|\left[\left(\left(\zeta_{2}+\zeta_{1}\right)\left(\rho_{1}\left\|e_{n+1}\right\|+\rho_{2}\left\|e_{n}\right\|_{1}^{2}\right)+M_{3}\left\|e_{n}\right\|_{1}^{2}\right)\right] d s \\
& +\left(\sum_{i=1}^{m-2} \tau_{i} e_{n+1}\left(\eta_{i}\right)\right)\left(\frac{-t}{2 q+p}+\frac{q+p}{p(2 q+p)}\right)+\left(\sum_{i=1}^{m-2} \sigma_{i} e_{n+1}\left(\eta_{i}\right)\right)\left(\frac{t}{2 q+p}+\frac{q}{p(2 q+p)}\right) \\
\leq & \int_{0}^{1}\left|G_{\zeta_{2}}(t, s)\right|\left(\zeta_{2}+\zeta_{1}\right)\left(\rho_{1}\left\|e_{n+1}\right\| d s+\int_{0}^{1}\left|G_{\zeta_{2}}(t, s)\right|\left(\rho_{2}\left(\zeta_{2}+\zeta_{1}\right)+M_{3}\right)\left\|e_{n}\right\|_{1}^{2}\right) d s \\
& +\left(\sum_{i=1}^{m-2} \tau_{i}+\sum_{i=1}^{m-2} \sigma_{i}\right)\left(\frac{p+q}{p(2 q+p)}\right) e_{n+1}\left(\eta_{i}\right) \\
\leq & \left(B+\left(\sum_{i=1}^{m-2} \tau_{i}+\sum_{i=1}^{m-2} \sigma_{i}\right)\left(\frac{p+q}{p(2 q+p)}\right)\right)\left\|e_{n+1}\right\|+A\left\|e_{n}\right\|_{1}^{2},
\end{aligned}
$$

where

$$
A=\left(\rho_{2}\left(\zeta_{2}+\zeta_{1}\right)+M_{3}\right) \max \int_{0}^{1}\left|G_{\zeta_{2}}(t, s)\right| d s, \quad B=\left(\zeta_{2}+\zeta_{1}\right) \rho_{1} \max \int_{0}^{1}\left|G_{\zeta_{2}}(t, s)\right| d s
$$

Taking the maximum over $[0,1]$ and then solving (3.43) for $\left\|e_{n+1}\right\|$, we obtain

$$
\left\|e_{n+1}\right\| \leq \frac{A}{1-B-\left(\sum_{i=1}^{m-2} \tau_{i}+\sum_{i=1}^{m-2} \sigma_{i}\right)(p+q / p(2 q+p))}\left\|e_{n}\right\|_{1}^{2} .
$$

Also, it follows from (3.33) that

$$
\left(e_{n+1}^{\prime} \mu(t)\right)^{\prime} \geq-M_{3}\left\|e_{n}\right\|_{1}^{2} \mu(t) \geq-M_{3} e^{\zeta_{2} t}\left\|e_{n}\right\|_{1}^{2}, \quad t \in[0,1] .
$$

Integrating (3.46) from 0 to $t$ and using $v_{n+1}^{\prime}(0) \geq(-1 / q) \sum_{i=1}^{m-2} \tau_{i} e_{n+1}\left(\eta_{i}\right)$ (from the boundary condition $\left.\left(p e_{n+1}(0)-q e_{n+1}^{\prime}(0)=\sum_{i=1}^{m-2} \tau_{i} e_{n+1}\left(\eta_{i}\right)\right)\right)$, we obtain

$$
e_{n+1}^{\prime}(t) \mu(t) \geq \frac{-1}{q} \sum_{i=1}^{m-2} \tau_{i} e_{n+1}\left(\eta_{i}\right)-\frac{M_{3}\left(e^{\zeta_{2} t}-1\right)}{\zeta_{2}}\left\|e_{n}\right\|_{1}^{2}
$$


which, in view of the fact $e^{-\zeta_{1} t}<\mu \leq e^{\zeta_{2} t}$ and (3.45), yields

$$
\begin{aligned}
e_{n+1}^{\prime}(t) \geq e^{\zeta_{1} t} & {\left[\left(\frac{-1}{q} \sum_{i=1}^{m-2} \tau_{i}\right)\left(\frac{A}{1-B-\left(\sum_{i=1}^{m-2} \tau_{i}+\sum_{i=1}^{m-2} \sigma_{i}\right)((p+q) / p(2 q+p))}\right)\right.} \\
& \left.-\frac{M_{3}\left(e^{\zeta_{2} t}-1\right)}{\zeta_{2}}\right]\left\|e_{n}\right\|_{1}^{2} \geq-\delta_{1}\left\|e_{n}\right\|_{1}^{2},
\end{aligned}
$$

where

$$
\begin{gathered}
\delta_{1}=\max \left\{e ^ { \zeta _ { 1 } t } \left[\left(\frac{1}{q} \sum_{i=1}^{m-2} \tau_{i}\right)\left(\frac{A}{1-B-\left(\sum_{i=1}^{m-2} \tau_{i}+\sum_{i=1}^{m-2} \sigma_{i}\right)((p+q) / p(2 q+p))}\right)\right.\right. \\
\left.\left.+\frac{M_{3}\left(e^{\zeta_{2} t}-1\right)}{\zeta_{2}}\right], t \in[0,1]\right\} .
\end{gathered}
$$

As $e_{n+1} \in C^{1}([0,1])$, there exists $\bar{t} \in(0,1)$ such that

$$
\begin{aligned}
e_{n+1}^{\prime}(\bar{t}) & =e_{n+1}(1)-e_{n+1}(0) \leq e_{n+1}(1) \\
& \leq \frac{1}{p} \sum_{i=1}^{m-2} \sigma_{i} e_{n+1}\left(\eta_{i}\right)-\frac{q}{p} e_{n+1}^{\prime}(1) \leq \frac{1}{p} \sum_{i=1}^{m-2} \sigma_{i}\left\|e_{n+1}\right\|+\frac{q \delta}{p}\left\|e_{n}\right\|_{1}^{2} \\
& \leq\left[\frac{A}{p\left[1-B-\left(\sum_{i=1}^{m-2} \sigma_{i}+\sum_{i=1}^{m-2} \tau_{i}\right)((p+q) / p(2 q+p))\right]} \sum_{i=1}^{m-2} \sigma_{i}+\frac{q \delta}{p}\right]\left\|e_{n}\right\|_{1}^{2} .
\end{aligned}
$$

Integrating (3.46) from $t$ to $\bar{t}(t \leq \bar{t})$ and using (3.50), we have

$$
\begin{aligned}
e_{n+1}^{\prime}(t) \leq e^{\zeta_{1} t}\left[\frac{e^{\zeta_{2} \bar{t}} A \sum_{i=1}^{m-2} \sigma_{i}}{p\left[1-B-\left(\sum_{i=1}^{m-2} \sigma_{i}+\sum_{i=1}^{m-2} \tau_{i}\right)((p+q) / p(2 q+p))\right]}\right. \\
\left.+\frac{q \delta}{p}+\frac{M_{3}\left(e^{\zeta_{2} \bar{t}}-e^{\zeta_{2} t}\right)}{\zeta_{2}}\right]\left\|e_{n}\right\|_{1}^{2} .
\end{aligned}
$$

Using (3.45) in (3.34), we obtain

$$
\left(e_{n+1}^{\prime}(t) \mu_{1}(t)\right)^{\prime} \leq \frac{\gamma A \mu_{1}(t)}{1-B-\left(\sum_{i=1}^{m-2} \sigma_{i}+\sum_{i=1}^{m-2} \tau_{i}\right)((p+q) / p(2 q+p))}\left\|e_{n}\right\|_{1}^{2},
$$


where $\mu_{1}(t)=e^{\int_{0}^{t} f_{x^{\prime}}\left(s, c_{3}, c_{4}\right) d s}$. Since $f_{x^{\prime}}$ is bounded on $[0,1] \times\left[\min _{t \in[0,1]} \alpha(t), \max _{t \in[0,1]} \beta(t)\right] \times$ $[-M, M]$, we can choose $\zeta_{3}, \zeta_{4}>0$ such that $-\zeta_{3} \leq f_{x^{\prime}\left(t, c_{3}, c_{4}\right)} \leq \zeta_{4}$ on $[0,1] \times\left[\min _{t \in[0,1]} \alpha(t)\right.$, $\left.\max _{t \in[0,1]} \beta(t)\right] \times[-M, M]$ and $e^{-\zeta_{3} t}<\mu_{1}(t) \leq e^{\zeta_{4} t}$ so that (3.52) takes the form

$$
\left(e_{n+1}^{\prime}(t) \mu_{1}(t)\right)^{\prime} \leq \frac{\gamma A e^{\zeta_{4} t}}{1-B-\left(\sum_{i=1}^{m-2} \sigma_{i}+\sum_{i=1}^{m-2} \tau_{i}\right)((p+q) / p(2 q+p))}\left\|e_{n}\right\|_{1}^{2} .
$$

Integrating (3.53) from $\bar{t}$ to $t(t \geq \bar{t})$, and using (3.51), we find that

$$
\begin{aligned}
e_{n+1}^{\prime}(t) \leq & \frac{1}{\mu_{1}(t)}\left[e_{n+1}^{\prime}(\bar{t}) \mu_{1}(\bar{t})+\frac{\gamma A\left(e^{\zeta_{4} t}-e^{\zeta_{4} \bar{t}}\right)}{L_{2}\left[1-B-\left(\sum_{i=1}^{m-2} \sigma_{i}+\sum_{i=1}^{m-2} \tau_{i}\right)((p+q) / p(2 q+p))\right]}\left\|e_{n}\right\|_{1}^{2}\right] \\
\leq & e^{\zeta_{3} t}\left[\frac{A e^{\zeta_{4} \bar{t}} \sum_{i=1}^{m-2} \sigma_{i}}{p\left[1-B-\left(\sum_{i=1}^{m-2} \sigma_{i}+\sum_{i=1}^{m-2} \tau_{i}\right)((p+q) / p(2 q+p))\right]}+\frac{q \delta e^{\zeta_{4} \bar{t}}}{p}\right. \\
& \left.+\frac{\gamma A\left(e^{\zeta_{4} t}-e^{\zeta_{4} \bar{t}}\right)}{\zeta_{4}\left[1-B-\left(\sum_{i=1}^{m-2} \sigma_{i}+\sum_{i=1}^{m-2} \tau_{i}\right)((p+q) / p(2 q+p))\right]}\right]\left\|e_{n}\right\|_{1}^{2} .
\end{aligned}
$$

Letting

$$
\begin{aligned}
& \delta_{2}=\max \left\{\operatorname { m a x } \left\{e ^ { \zeta _ { 1 } t } \left[\frac{e^{\zeta_{2} \bar{t}} A \sum_{i=1}^{m-2} \sigma_{i}}{p\left[1-B-\left(\sum_{i=1}^{m-2} \sigma_{i}+\sum_{i=1}^{m-2} \tau_{i}\right)((p+q) / p(2 q+p))\right]}\right.\right.\right. \\
& \left.\left.+\frac{q \delta}{p}+\frac{M_{3}\left(e^{\zeta_{2} \bar{t}}-e^{\zeta_{2} t}\right)}{\zeta_{2}}\right], t \in[0, \bar{t}]\right\} \\
& \max \left\{e ^ { \zeta _ { 3 } t } \left[\frac{A e^{\zeta_{4} \bar{t}} \sum_{i=1}^{m-2} \sigma_{i}}{p\left[1-B-\left(\sum_{i=1}^{m-2} \sigma_{i}+\sum_{i=1}^{m-2} \tau_{i}\right)((p+q) / p(2 q+p))\right]}+\frac{q \delta e^{\zeta_{4} \bar{t}}}{p}\right.\right. \\
& \left.\left.\left.+\frac{\gamma A\left(e^{\zeta_{4} t}-e^{\zeta_{4} \bar{t}}\right)}{\zeta_{4}\left[1-B-\left(\sum_{i=1}^{m-2} \sigma_{i}+\sum_{i=1}^{m-2} \tau_{i}\right)((p+q) / p(2 q+p))\right]}\right], t \in[\bar{t}, 1]\right\}\right\},
\end{aligned}
$$


it follows from (3.51) and (3.54) that

$$
e_{n+1}^{\prime}(t) \leq \delta_{2}\left\|e_{n}\right\|_{1}^{2}
$$

Hence, from (3.48) and (3.56), it follows that

$$
\left\|e_{n+1}^{\prime}\right\| \leq \delta_{3}\left\|e_{n}\right\|_{1}^{2}
$$

where $\delta_{3}=\max \left\{\delta_{1}, \delta_{2}\right\}$. From (3.45) and (3.57) with

$$
Q=\frac{A}{\left[1-B-\left(\sum_{i=1}^{m-2} \sigma_{i}+\sum_{i=1}^{m-2} \tau_{i}\right)((p+q) / p(2 q+p))\right]}+\delta_{3}
$$

we obtain

$$
\left\|e_{n+1}\right\|_{1}=\left\|e_{n+1}\right\|+\left\|v_{n+1}^{\prime}\right\| \leq Q\left\|e_{n}\right\|_{1}^{2} .
$$

This proves the quadratic convergence in $C^{1}$ norm.

Example 3.3. Consider the boundary value problem

$$
\begin{gathered}
-x^{\prime \prime}=-\frac{1}{720} t e^{x}-\frac{1}{35}(x-1)-\frac{t\left(x^{\prime}\right)^{2}}{16\left(1+\left(x^{\prime}\right)^{2}\right)}, \quad t \in[0,1] \\
\frac{5}{4} x(0)-\frac{11}{20} x^{\prime}(0)=\frac{1}{7} x\left(\frac{3}{4}\right)+\frac{1}{9} x\left(\frac{4}{5}\right), \quad \frac{5}{4} x(1)+\frac{11}{20} x^{\prime}(1)=\frac{1}{3} x\left(\frac{3}{4}\right) .
\end{gathered}
$$

Let $\alpha(t)=0$ and $\beta(t)=1+t$ be, respectively, lower and upper solutions of (3.60). Clearly $\alpha(t)$ and $\beta(t)$ are not the solutions of (3.60) and $\alpha(t)<\beta(t), t \in[0,1]$. Also, the assumptions of Theorem 3.1 are satisfied. Thus, the conclusion of Theorem 3.1 applies to the problem (3.60).

\section{Acknowledgment}

The author is grateful to the referees and professor G. Infante for their valuable suggestions and comments that led to the improvement of the original paper. 


\section{References}

[1] G. S. Ladde, V. Lakshmikantham, and A. S. Vatsala, Monotone Iterative Techniques for Nonlinear Differential Equations, Monographs, Advanced Texts and Surveys in Pure and Applied Mathematics, 27, Pitman, Boston, Mass, USA, 1985.

[2] J. J. Nieto, Y. Jiang, and Y. Jurang, "Monotone iterative method for functional-differential equations," Nonlinear Analysis: Theory, Methods \& Applications, vol. 32, no. 6, pp. 741-747, 1998.

[3] A. S. Vatsala and J. Yang, "Monotone iterative technique for semilinear elliptic systems," Boundary Value Problems, vol. 2005, no. 2, pp. 93-106, 2005.

[4] Z. Drici, F. A. McRae, and J. V. Devi, "Monotone iterative technique for periodic boundary value problems with causal operators," Nonlinear Analysis: Theory, Methods \& Applications, vol. 64, no. 6, pp. 1271-1277, 2006.

[5] D. Jiang, J. J. Nieto, and W. Zuo, "On monotone method for first and second order periodic boundary value problems and periodic solutions of functional differential equations," Journal of Mathematical Analysis and Applications, vol. 289, no. 2, pp. 691-699, 2004.

[6] J. J. Nieto and R. Rodríguez-López, "Monotone method for first-order functional differential equations," Computers \& Mathematics with Applications, vol. 52, no. 3-4, pp. 471-484, 2006.

[7] B. Ahmad and S. Sivasundaram, "The monotone iterative technique for impulsive hybrid set valued integro-differential equations," Nonlinear Analysis: Theory, Methods E Applications, vol. 65, no. 12, pp. 2260-2276, 2006.

[8] A. Cabada and J. J. Nieto, "Rapid convergence of the iterative technique for first order initial value problems," Applied Mathematics and Computation, vol. 87, no. 2-3, pp. 217-226, 1997.

[9] V. Lakshmikantham and J. J. Nieto, "Generalized quasilinearization for nonlinear first order ordinary differential equations," Nonlinear Times and Digest, vol. 2, no. 1, pp. 1-9, 1995.

[10] R. E. Bellman and R. E. Kalaba, Quasilinearization and Nonlinear Boundary-Value Problems, vol. 3 of Modern Analytic and Computional Methods in Science and Mathematics, American Elsevier, New York, NY, USA, 1965.

[11] V. Lakshmikantham and A. S. Vatsala, Generalized Quasilinearization for Nonlinear Problems, vol. 440 of Mathematics and Its Applications, Kluwer Academic Publishers, Dordrecht, The Netherlands, 1998.

[12] A. Cabada and J. J. Nieto, "Quasilinearization and rate of convergence for higher-order nonlinear periodic boundary-value problems," Journal of Optimization Theory and Applications, vol. 108, no. 1, pp. 97-107, 2001.

[13] B. Ahmad, J. J. Nieto, and N. Shahzad, "The Bellman-Kalaba-Lakshmikantham quasilinearization method for Neumann problems," Journal of Mathematical Analysis and Applications, vol. 257, no. 2, pp. 356-363, 2001.

[14] P. W. Eloe and Y. Gao, "The method of quasilinearization and a three-point boundary value problem," Journal of the Korean Mathematical Society, vol. 39, no. 2, pp. 319-330, 2002.

[15] F. T. Akyildiz and K. Vajravelu, "Existence, uniqueness, and quasilinearization results for nonlinear differential equations arising in viscoelastic fluid flow," Differential Equations \& Nonlinear Mechanics, vol. 2006, Article ID 71717, 9 pages, 2006.

[16] B. Ahmad, "A quasilinearization method for a class of integro-differential equations with mixed nonlinearities," Nonlinear Analysis: Real World Applications, vol. 7, no. 5, pp. 997-1004, 2006.

[17] R. Krivec and V. B. Mandelzweig, "Quasilinearization method and WKB," Computer Physics Communications, vol. 174, no. 2, pp. 119-126, 2006.

[18] P. Amster and P. De Nápoli, "A quasilinearization method for elliptic problems with a nonlinear boundary condition," Nonlinear Analysis: Theory, Methods \& Applications, vol. 66, no. 10, pp. 2255-2263, 2007.

[19] B. Ahmad, A. Alsaedi, and B. S. Alghamdi, "Analytic approximation of solutions of the forced Duffing equation with integral boundary conditions," Nonlinear Analysis: Real World Applications, vol. 9, no. 4, pp. 1727-1740, 2008.

[20] B. Ahmad and J. J. Nieto, "Existence and approximation of solutions for a class of nonlinear impulsive functional differential equations with anti-periodic boundary conditions," Nonlinear Analysis: Theory, Methods \& Applications, vol. 69, no. 10, pp. 3291-3298, 2008.

[21] A. V. Bicadze and A. A. Samarskiı̌, "Some elementary generalizations of linear elliptic boundary value problems," Doklady Akademii Nauk SSSR, vol. 185, pp. 739-740, 1969.

[22] C. P. Gupta, "A second order $m$-point boundary value problem at resonance," Nonlinear Analysis: Theory, Methods E Applications, vol. 24, no. 10, pp. 1483-1489, 1995. 
[23] R. Ma, "Multiple positive solutions for nonlinear $m$-point boundary value problems," Applied Mathematics and Computation, vol. 148, no. 1, pp. 249-262, 2004.

[24] P. W. Eloe and B. Ahmad, "Positive solutions of a nonlinear $n$th order boundary value problem with nonlocal conditions," Applied Mathematics Letters, vol. 18, no. 5, pp. 521-527, 2005.

[25] J. R. L. Webb and G. Infante, "Positive solutions of nonlocal boundary value problems: a unified approach," Journal of the London Mathematical Society, vol. 74, no. 3, pp. 673-693, 2006.

[26] R. A. Khan, "Generalized approximations and rapid convergence of solutions of $m$-point boundary value problems," Applied Mathematics and Computation, vol. 188, no. 2, pp. 1878-1890, 2007.

[27] M. Pei and S. K. Chang, "The generalized quasilinearization method for second-order three-point boundary value problems," Nonlinear Analysis: Theory, Methods \& Applications, vol. 68, no. 9, pp. 27792790, 2008.

[28] M. Pei and S. K. Chang, "A quasilinearization method for second-order four-point boundary value problems," Applied Mathematics and Computation, vol. 202, no. 1, pp. 54-66, 2008.

[29] B. Ahmad, "Approximation of solutions of the forced Duffing equation with m-point boundary conditions," Communications in Applied Analysis, vol. 13, no. 1, pp. 11-20, 2009.

[30] L. Wang, M. Pei, and W. Ge, "Existence and approximation of solutions for nonlinear second-order four-point boundary value problems," Mathematical and Computer Modelling, vol. 50, no. 9-10, pp. 1348-1359, 2009.

[31] Y.-K. Chang, J. J. Nieto, and W.-S. Li, “On impulsive hyperbolic differential inclusions with nonlocal initial conditions," Journal of Optimization Theory and Applications, vol. 140, no. 3, pp. 431-442, 2009.

[32] J. R. Graef and J. R. L. Webb, "Third order boundary value problems with nonlocal boundary conditions," Nonlinear Analysis: Theory, Methods \& Applications, vol. 71, no. 5-6, pp. 1542-1551, 2009.

[33] M. Grossinho and F. M. Minhós, "Upper and lower solutions for higher order boundary value problems," Nonlinear Studies, vol. 12, no. 2, pp. 165-176, 2005. 